(C) 2016 IEEE. Personal use of this material is permitted. Permission from IEEE must be obtained for all other uses, in any current or future media, including reprinting/republishing this material for advertising or promotional purposes, creating new collective works, for resale or redistribution to servers or lists, or reuse of any copyrighted component of this work in other works. 


\section{Planning Wrench-Feasible Motions for Cable-driven Hexapods}

Oriol Bohigas, Montserrat Manubens, and Lluís Ros

\begin{abstract}
Motion paths of cable-driven hexapods must carefully be planned to ensure that the lengths and tensions of all cables remain within acceptable limits, for a given wrench applied to the platform. The cables cannot go slack -to keep the control of the robot- nor excessively tight -to prevent cable breakage- even in the presence of bounded perturbations of the wrench. This paper proposes a path planning method that accommodates such constraints simultaneously. Given two configurations of the robot, the method attempts to connect them through a path that, at any point, allows the cables to counteract any wrench lying in a predefined uncertainty region. The configuration space, or C-space for short, is placed in correspondence with a smooth manifold, which facilitates the definition of a continuation strategy to search this space systematically from one configuration, until the second configuration is found, or path non-existence is proved by exhaustion of the search. The force Jacobian is full rank everywhere on the C-space, which implies that the computed paths will naturally avoid crossing the forward singularity locus of the robot. The adjustment of tension limits, moreover, allows to maintain a meaningful clearance relative to such locus. The approach is applicable to compute paths subject to geometric constraints on the platform pose, or to synthesize free-flying motions in the full six-dimensional C-space. Experiments are included that illustrate the performance of the method in a real prototype.
\end{abstract}

Index Terms-Cable-driven hexapod, wrench-feasible C-space, higherdimensional continuation, singularity-free path planning.

\section{INTRODUCTION}

In recent years, cable-driven parallel robots have been increasingly studied and applied to more and more relevant tasks, such as manipulation of heavy loads [1, 2], high-precision positioning [3], monitoring of aquatic environments [4], automated construction of civil structures [5], rescue systems [6], or motion simulators [7]. Among them, hexapodal ones stand out for their simplicity and extensive use (Fig. 1), especially after the long-term effort on the NIST Robocrane and its derived applications [3, 8]-[10]. They involve the minimum number of cables and motors to fully govern a load in 6D under gravity, resulting in simple robotic cranes for precise manipulation that can even be made mobile by attaching vehicles to the feet [11]. These advantages, together with the fact that they can easily achieve larger workspaces than their counterparts with rigid-limb legs, make cable-driven hexapods energy-efficient and appropriate to maneuver heavy objects in position and orientation. However, additional constraints apply: their cables can pull but are unable to push the platform, which obliges to keep the cable tensions positive during normal operation.

The C-space of a cable-driven hexapod is limited by a number of hypersurfaces corresponding to configurations where the tension of some cable is either zero, for which the cable goes slack and control of one degree of freedom is lost (see [12] for a dramatic example), or goes to infinity, which indicates that the mechanism is in a singular configuration and the cable can break [13]. In practice, it is important to prevent both extreme situations and ensure that the cables work within a range of admissible tensions, for a given

Oriol Bohigas, Montserrat Manubens, and Lluís Ros are with the Kinematics and Robot Design Group at Institut de Robòtica i Informàtica Industrial, CSIC-UPC, Llorens Artigas 4-6, 08028 Barcelona, Spain (emails: \{obohigas,mmanuben,lros\}@iri.upc.edu).

This work has been partially supported by the Spanish Ministry of Economy under project DPI2014-57220-C2-2-P, by the CSIC project 201250E026, and by a Juan de la Cierva fellowship supporting Montserrat Manubens.

The paper has supplementary downloadable material that includes a video of the experiments in Fig. 9. platform wrench subject to bounded perturbations in all directions. After [14] and [15], the configurations fulfilling this condition are said to be wrench-feasible.

Several methods have been proposed for the determination of wrench-feasible workspaces of cable-driven parallel robots [13, 15][18], but the problem of planning paths between given configurations has received little attention comparatively. Algorithms indeed exist that try to avoid the singular configurations where the leg forces tend to infinity, but they are mainly tailored to parallel robots with rigid UPS legs [19]-[22], and their application to cable-driven hexapods is not straightforward because they do not account for the positivity constraint on the leg tensions. Moreover, these algorithms measure the clearance of the path relative to the singularity locus using the determinant or the condition number of the force Jacobian matrix, which, as noted in [23], lack physical significance. While some path planning approaches apply to cable-driven hexapods [24]-[26], the path they compute is evaluated for feasibility at discrete points only, so a method that guarantees the fulfillment of all the constraints along the whole path is still lacking.

The planning method presented in this paper is aimed at covering such gap. It was preliminarly introduced in [27] and it is now presented with thorough detail and illustrative experiments. The method relies on defining a system of equations whose solution manifold corresponds to the six-dimensional wrench-feasible C-space of the hexapod, so that maneuvering through such manifold guarantees singularity avoidance at all times, while maintaining cable tensions and lengths within their allowable positive bounds (Sections II and III). This manifold, as well as any of its subsets defined by motion constraints arising in many applications, are found to be smooth everywhere, which is key to define a continuation method able to explore the C-space systematically from one configuration, until a goal configuration is found, or path non-existence is proved at the resolution of the search (Section IV). The method has been implemented and validated in several test cases, and with experiments in a real prototype (Section V). Its main strengths and points for future attention have been identified as well (Section VI).

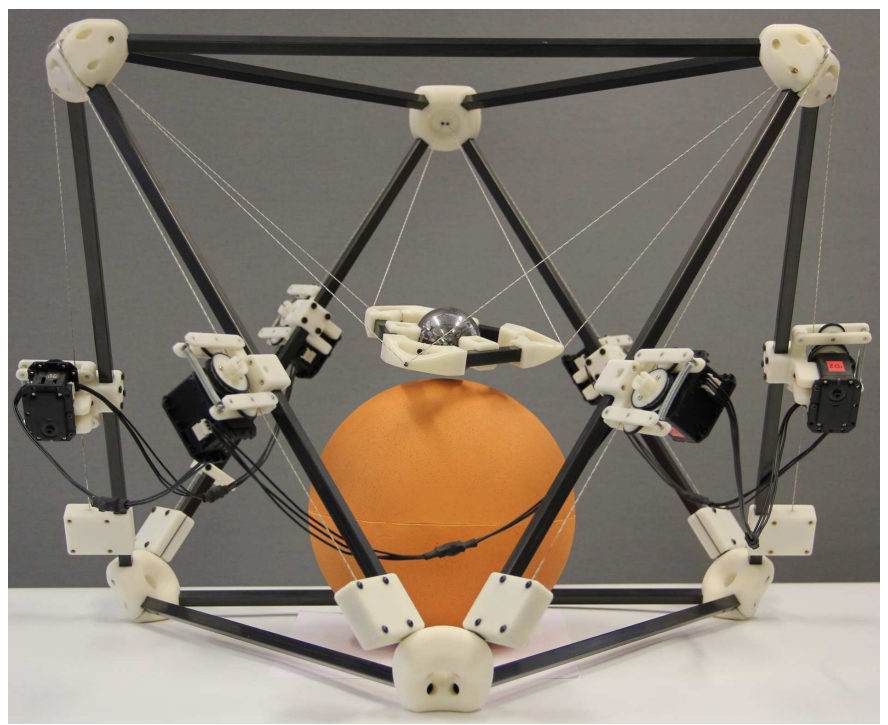

Fig. 1. A cable-driven hexapod consists of a moving platform connected to a fixed base by means of six cables of variable (actuated) length. The platform is maintained in a stable position due to the action of gravity. This prototype was constructed at Institut de Robòtica i Informàtica Industrial to test the planning method proposed (Section V). 


\section{Preliminaries}

A cable-driven hexapod consists of a moving platform suspended from a fixed base by means of six cables winding around independent winches (Fig. 2). The cables are herein assumed to be of neglectable mass and elasticity. By actuating the winch drives, the cable lengths $d_{i}$ can be varied within prescribed limits $\left(\underline{d_{i}}, \overline{d_{i}}\right)$, with $\underline{d_{i}}>0$, which allows a full control of the six degrees of freedom of the platform within a given workspace. The $\mathrm{C}$-space of such a robot, and the planning problem confronted, can be defined as follows.

Consider fixed and moving reference frames $\mathcal{F}_{1}$ and $\mathcal{F}_{2}$, respectively attached to the base and platform links, centered in $O$ and $P$ (Fig. 2). Let $\boldsymbol{p}$ and $\boldsymbol{a}_{i}$ be the position vectors of $P$ and $A_{i}$ relative to $\mathcal{F}_{1}$, and $\boldsymbol{b}_{i}$ be the position vector of $B_{i}$ relative to $\mathcal{F}_{2}$. We can represent any platform configuration by the pair $\boldsymbol{q}=(\boldsymbol{p}, \boldsymbol{R}) \in S E(3)=\mathbb{R}^{3} \times S O(3)$, subject to the constraints

$$
\begin{gathered}
\boldsymbol{d}_{i}=\boldsymbol{p}+\boldsymbol{R} \boldsymbol{b}_{i}-\boldsymbol{a}_{i}, \\
d_{i}^{2}=\boldsymbol{d}_{i}^{\top} \boldsymbol{d}_{i}, \\
\underline{d_{i}}<d_{i}<\overline{d_{i}},
\end{gathered}
$$

for $i=1, \ldots, 6$, where $\boldsymbol{R}$ is the $3 \times 3$ rotation matrix that provides the orientation of $\mathcal{F}_{2}$ relative to $\mathcal{F}_{1}$. While Eqs. (1) and (2) make the cable lengths $d_{i}$ explicit in terms of $\boldsymbol{p}$ and $\boldsymbol{R}$, the inequalities in (3) constrain such lengths to lie in $\left(\underline{d_{i}}, \overline{d_{i}}\right)$.

The $\boldsymbol{R}$ matrix in Eq. (1) is assumed to be expressed as a function of $\boldsymbol{\tau}$, a tuple of any three angles parameterizing $S O(3)$, such as Euler angles under any convention, or tilt-and-torsion angles [28]. This allows for an easy formulation of planning problems in constantangle slices of $S E(3)$, which are useful in parallel kinematic machines [28], and avoids the treatment of additional constraints needed in non-minimal representations of the rotation group. Although we then introduce representation singularities relative to the angles of choice [29, page 31], this will not be problematic because the smoothness properties required to solve our planning problem will remain unaltered.

In practice, any configuration must also be wrench-feasible, i.e., it must allow the platform to equilibrate any external wrench $\hat{\boldsymbol{w}}$ acting on it, subject to lie inside a prescribed, bounded region $\mathcal{K} \subset \mathbb{R}^{6}$. The coordinates of $\hat{\boldsymbol{w}}$ are assumed to be given in the usual screwtheoretic form [30], i.e., the first three components provide the net force on the platform, and the last three ones give the net moment relative to $O$. The significance of $\mathcal{K}$ depends on the particular context

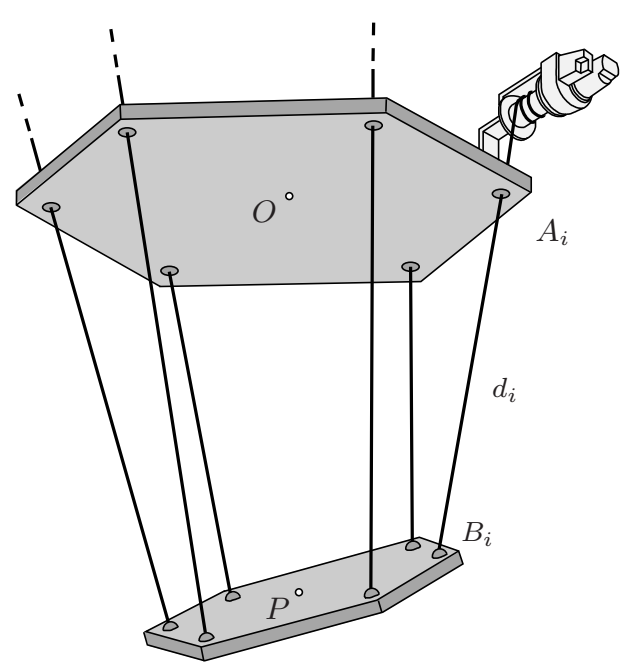

Fig. 2. Kinematic structure of a cable-driven hexapod. of application. In payload transportation, for instance, $\mathcal{K}$ may be given by the gravitational wrench acting on the platform and slight perturbations introduced by inertia forces or external agents like the wind. In contact situations, $\mathcal{K}$ might further depend on the contact wrench applied by the environment, which is in general subject to six-dimensional uncertainty. Specifically, the wrench-feasibility requirement on a given $\boldsymbol{q}$ implies that for each $\hat{\boldsymbol{w}} \in \mathcal{K}$ there must be a vector of admissible cable tensions

$$
\boldsymbol{f}=\left[f_{1}, \ldots, f_{6}\right]^{\top} \in \mathcal{D}=\left(\underline{f_{1}}, \overline{f_{1}}\right) \times \ldots \times\left(\underline{f_{6}}, \overline{f_{6}}\right)
$$

satisfying

$$
\boldsymbol{J} \boldsymbol{f}=\hat{\boldsymbol{w}},
$$

where $\left(f_{i}, \overline{f_{i}}\right)$ is the range of positive tensions that can be resisted by the $i$ th cable, and $\boldsymbol{J}$ is the $6 \times 6$ force Jacobian of the robot. $\boldsymbol{J}$ is a function of $\boldsymbol{q}$ and takes the form

$$
\boldsymbol{J}=\left[\begin{array}{ccc}
\boldsymbol{u}_{1} & \cdots & \boldsymbol{u}_{6} \\
\boldsymbol{a}_{1} \times \boldsymbol{u}_{1} & \cdots & \boldsymbol{a}_{6} \times \boldsymbol{u}_{6}
\end{array}\right],
$$

in frame $\mathcal{F}_{1}$, where $\boldsymbol{u}_{i}=\boldsymbol{d}_{i} / d_{i}$ [30].

For ease of manipulation, $\mathcal{K}$ will be assumed to be a sixdimensional ellipsoid centered in $\hat{\boldsymbol{w}}_{0}$, defined implicitly by the inequality

$$
\left(\hat{\boldsymbol{w}}-\hat{\boldsymbol{w}}_{0}\right)^{\top} \boldsymbol{E}\left(\hat{\boldsymbol{w}}-\hat{\boldsymbol{w}}_{0}\right) \leq 1,
$$

where $\boldsymbol{E}$ is a $6 \times 6$ positive-definite symmetric matrix. This ellipsoid can be constructed by propagating known bounds on other variables related to $\hat{\boldsymbol{w}}$, using the tools of an ellipsoidal calculus for example [31]. In [32], we explain how to obtain $\hat{\boldsymbol{w}}_{0}$ and $\boldsymbol{E}$ in typical situations, and show that $\hat{\boldsymbol{w}}_{0}$ and $\boldsymbol{E}$ are a function of $\boldsymbol{q}$ in general.

We can now define the $\mathrm{C}$-space of the manipulator, $\mathcal{C}$, as the set of wrench-feasible configurations $\boldsymbol{q} \in S E(3)$ that satisfy Eqs. (1)(3) for $i=1, \ldots, 6$. The planning problem we confront, thus, boils down to computing a path joining two given configurations of $\mathcal{C}, \boldsymbol{q}_{s}$ and $\boldsymbol{q}_{g}$; i.e., a continuous map

$$
\boldsymbol{\mu}:[0,1] \longrightarrow \mathcal{C}
$$

such that $\boldsymbol{\mu}(0)=\boldsymbol{q}_{s}$ and $\boldsymbol{\mu}(1)=\boldsymbol{q}_{g}$. To tackle this problem, we next define a smooth manifold suitable to navigate $\mathcal{C}$ by numerical continuation [33].

\section{The NAVIGATION MANifold}

\section{A. A Characterization of $\mathcal{C}$}

For a given configuration $\boldsymbol{q}$ and a wrench $\hat{\boldsymbol{w}}_{0}$ applied to the platform, let $\boldsymbol{f}_{0}$ be the vector of cable tensions corresponding to $\hat{\boldsymbol{w}}_{0} \in \mathcal{K}$, which satisfies

$$
\boldsymbol{J} \boldsymbol{f}_{0}=\hat{\boldsymbol{w}}_{0} .
$$

By noting that $\boldsymbol{J}\left(\boldsymbol{f}-\boldsymbol{f}_{0}\right)=\hat{\boldsymbol{w}}-\hat{\boldsymbol{w}}_{0}$, it is easy to see that the set $\mathcal{L}$ of cable tensions $\boldsymbol{f}$ corresponding to all wrenches $\hat{\boldsymbol{w}} \in \mathcal{K}$ is the ellipsoid given by

$$
\left(\boldsymbol{f}-\boldsymbol{f}_{0}\right)^{\top} \boldsymbol{B}\left(\boldsymbol{f}-\boldsymbol{f}_{0}\right) \leq 1
$$

where $\boldsymbol{B}=\boldsymbol{J}^{\top} \boldsymbol{E} \boldsymbol{J}$. This ellipsoid may be bounded in all directions or unbounded in some, depending on whether $\operatorname{det}(\boldsymbol{J}) \neq 0$ or not. However, [32, Chapter 6] shows that $\boldsymbol{J}$ is non-singular for all $\boldsymbol{q} \in \mathcal{C}$, so that $\mathcal{L}$ will always be a bounded ellipsoid in our case (Fig. 3). It is worth to see here that, since $J$ is full rank for all $q \in \mathcal{C}$, the navigation of $\mathcal{C}$ implicitly avoids the singular configurations of the platform. Thus, the control issues related to such configurations (due to output velocity indetermination and platform shakiness [34]) will not be encountered during the execution of the obtained path. 


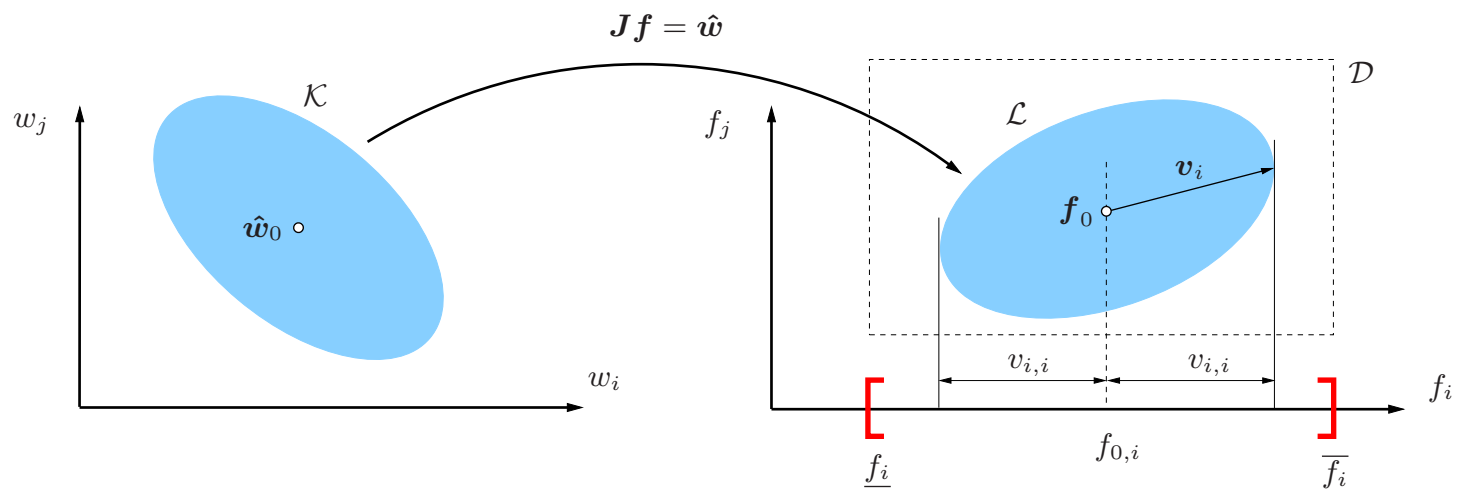

Fig. 3. The mapping $\boldsymbol{J} \boldsymbol{f}=\hat{\boldsymbol{w}}$ is used to transform the wrench ellipsoid $\mathcal{K}$ into the cable tension ellipsoid $\mathcal{F}$. The arrow in the figure indicates the transformation from the former ellipsoid to the latter. The vector $\boldsymbol{v}_{i}$ provides the maximum and minimum values of $f_{i}$ within $\mathcal{F}$.

Now, for $\boldsymbol{q}$ to be wrench-feasible, we must have $\mathcal{L} \subseteq \mathcal{D}$, which can be checked as follows. Consider the vector $\boldsymbol{v}_{i}$ that gives the offset from the center of $\mathcal{L}$ to the point of $\mathcal{L}$ attaining the largest $f_{i}$ value, $\boldsymbol{f}_{0}+\boldsymbol{v}_{i}$ (Fig. 3). Symmetrically, $\boldsymbol{f}_{0}-\boldsymbol{v}_{i}$ is the point of $\mathcal{L}$ with the smallest $f_{i}$ value. Using Lagrange multipliers, one can see that $\boldsymbol{v}_{i}$ is the unique vector satisfying

$$
\begin{gathered}
\boldsymbol{v}_{i}^{\top} \boldsymbol{B} \boldsymbol{v}_{i}=1, \\
\boldsymbol{B}^{i} \boldsymbol{v}_{i}=\mathbf{0}, \\
v_{i, i}>0,
\end{gathered}
$$

where $\boldsymbol{B}^{i}$ is the matrix $\boldsymbol{B}$ with its $i$ th row removed, and $v_{i, i}$ is the $i$-th component of $\boldsymbol{v}_{i}$. Observe that if $\boldsymbol{J}$ is non-singular, then both $\boldsymbol{B}$ and $\boldsymbol{B}^{i}$ are full row rank, and certainly there is exactly one vector $\boldsymbol{v}_{i}$ satisfying Eqs. (5)-(7). Using this vector, we can say that if $\operatorname{det}(\boldsymbol{J}) \neq 0$, then $\mathcal{L}$ is contained in $\mathcal{D}$ whenever

$$
\begin{aligned}
& f_{0, i}-v_{i, i}>\underline{f_{i},}, \\
& f_{0, i}+v_{i, i}<\overline{\overline{f_{i}}},
\end{aligned}
$$

for $i=1, \ldots, 6$. As a result, $\mathcal{C}$ can be characterized as the set of points $\boldsymbol{q} \in S E(3)$ satisfying Eqs. (1)-(9) for some value of the variables $\boldsymbol{d}_{i}, d_{i}, \boldsymbol{f}_{0}$ and $\boldsymbol{v}_{i}$.

\section{B. Conversion into Equality Form}

Continuation methods are, by design, aimed at tracing solution sets of systems of equations, not inequalities [33]. To define a continuation-based path planning strategy, we thus need to convert Eqs. (3), and (7)-(9) into equality form. To this end, note from Fig. 4 (a) that we can replace Eq. (3) by

$$
\begin{gathered}
\left(d_{i}-\underline{d_{i}}\right) \cdot\left(\overline{d_{i}}-d_{i}\right) \cdot g_{i}=1, \\
g_{i}>0,
\end{gathered}
$$

where $g_{i}$ is a newly-defined auxiliary variable. In apparence, we have not skipped the use of inequalities with this change, but from the graph of Fig. 4 (a) we see that if a configuration $\boldsymbol{q}$ corresponds to a value $d_{i}=a \in\left(d_{i}, \overline{d_{i}}\right)$, then any other configuration found from $\boldsymbol{q}$ by continuation subject to Eq. (10) will always satisfy $d_{i}<d_{i}<\overline{d_{i}}$. In other words, the constraint $g_{i}>0$ can be neglected under such a continuation scheme.

Similarly, Eqs. (8) and (9) can be replaced by

$$
\begin{gathered}
\left(f_{0, i}-v_{i, i}-\underline{f_{i}}\right) \cdot s_{i}=1, \\
\left(\overline{f_{i}}-f_{0, i}-v_{i, i}\right) \cdot t_{i}=1, \\
s_{i}>0, \quad t_{i}>0,
\end{gathered}
$$

(a)

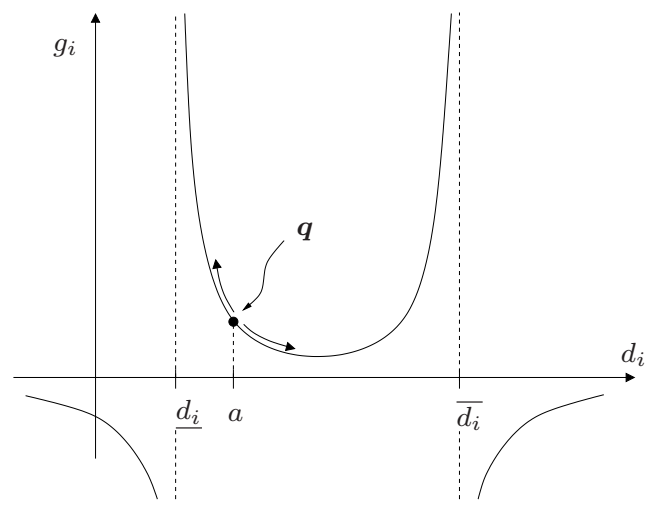

(b)

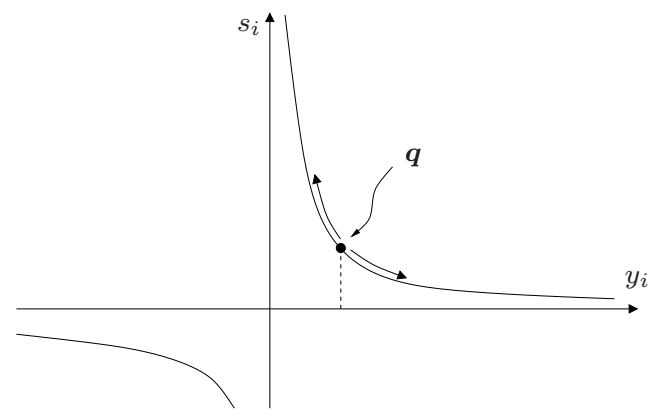

Fig. 4. (a) The graph of Eq. (10) shows that if we explore the solution set of Eq. (10) by continuation from some $\boldsymbol{q} \in \mathcal{C}$ corresponding to $d_{i}=a$, the constraints $d_{i}<d_{i}<\overline{d_{i}}$ will always be satisfied. (b) The graph of $y_{i} s_{i}=1$, where $y_{i}=f_{0, i}-v_{i, i}-\underline{f_{i}}$, shows that the same applies to Eq. (11).

where $s_{i}$ and $t_{i}$ play a role analogous to that of $g_{i}$ in Eq. (10). From the graph in Fig. 4 (b), for example, it is clear that the quantity $y_{i}=f_{0, i}-v_{i, i}-\underline{f_{i}}$ will remain positive, and hence $f_{0, i}-v_{i, i}>\underline{f_{i}}$, when marching continuously from a given $\boldsymbol{q}$ with $y_{i}>0$. The same argument applies to Eq. (12), so we can replace Eqs. (8) and (9) by Eqs. (11) and (12), neglecting the constraints $s_{i}>0$ and $t_{i}>0$ during the continuation scheme.

Finally, Eq. (7) can be directly neglected, because $v_{i, i} \neq 0$ for all $i$ on any vector satisfying Eqs. (5) and (6). Certainly, observe that $\boldsymbol{B} \boldsymbol{v}_{i}$ is all zeros except in its $i$-th component due to Eq. (6). If it were $v_{i, i}=0$ for some $i$, this would imply $\boldsymbol{v}_{i}^{\top} \boldsymbol{B} \boldsymbol{v}_{i}=0$, contradicting Eq. (5). Therefore, if our continuation method starts from a value of $\boldsymbol{v}_{i}$ with $v_{i, i}>0$, and it is compliant with Eqs. (5) and (6), Eq. (7) will be naturally fulfilled. 


\section{The Manifold and its Properties}

The system formed by Eqs. (1)-(2), (4)-(6), and (10)-(12) can be compactly written as

$$
\boldsymbol{F}(\boldsymbol{x})=\mathbf{0},
$$

where $\boldsymbol{x}$ refers to a tuple encompassing all of its variables: $\boldsymbol{p}, \boldsymbol{\tau}, \boldsymbol{d}_{i}, d_{i}, \boldsymbol{f}_{0}, \boldsymbol{v}_{i}, g_{i}, s_{i}$, and $t_{i}$, for $i=1, \ldots, 6$. The solution set of this system,

$$
\mathcal{M}=\{\boldsymbol{x}: \boldsymbol{F}(\boldsymbol{x})=\mathbf{0}\},
$$

will be called the navigation manifold hereafter, because it has the necessary properties to connect $\boldsymbol{q}_{s}$ and $\boldsymbol{q}_{g}$ by numerical continuation.

To see this point, consider the subset $\mathcal{M}^{+} \subset \mathcal{M}$ formed by the points $\boldsymbol{x}$ for which $g_{i}>0, s_{i}>0, t_{i}>0$, and $v_{i, i}>0$. Clearly, $\mathcal{C}$ and $\mathcal{M}^{+}$are in correspondence. A point $\boldsymbol{q}$ belongs to $\mathcal{C}$ if, and only if, it has a corresponding point $\boldsymbol{x} \in \mathcal{M}^{+}$, and any continuous path in $\mathcal{C}$ will also be represented by a continuous path in $\mathcal{M}^{+}$, and vice versa. Thus, the original problem of computing a path of $\mathcal{C}$ connecting $\boldsymbol{q}_{s}$ and $\boldsymbol{q}_{g}$ can be reduced to that of computing a path in $\mathcal{M}^{+}$connecting points $\boldsymbol{x}_{s}$ and $\boldsymbol{x}_{g}$ of $\mathcal{M}^{+}$corresponding to $\boldsymbol{q}_{s}$ and $\boldsymbol{q}_{g}$. However, since $g_{i}, s_{i}, t_{i}$, and $v_{i, i}$ never vanish on $\mathcal{M}$ (Section III-B), $\mathcal{M}^{+}$and its complement $\mathcal{M} \backslash \mathcal{M}^{+}$are disconnected, and if we try to connect $\boldsymbol{x}_{s}$ to $\boldsymbol{x}_{g}$ by continuation on $\mathcal{M}$, we will be moving through $\mathcal{M}^{+}$ actually. Therefore, for the purpose of this paper, we will only need $\mathcal{M}$ and Eq. (13) hereafter.

$\mathcal{M}$ is six-dimensional, and [32, Chapter 6] further proves that it is smooth everywhere, so that every point $\boldsymbol{x}$ has a well-defined tangent space $T_{\boldsymbol{x}} \mathcal{M}$. This greatly simplifies the definition of a continuation method to connect $\boldsymbol{x}_{s}$ and $\boldsymbol{x}_{g}$, because no bifurcations, sharpnesses, or dimension changes will be found along the way, avoiding the need of elaborate branch-switching procedures [35].

It is worth mentioning that in many applications (such as in painting, polishing, or cleaning of ship hulls, or building façades) the platform is further confined to move within a lower-dimensional subset of $\mathcal{C}$ defined by geometric or contact constraints on its pose. As exemplified in Section V-B, we can directly add such constraints to Eq. (13) if we wish, written either in the parametric form

$$
\left[\begin{array}{l}
p \\
\tau
\end{array}\right]=\Omega(\lambda)
$$

where $\Omega$ is an arbitrary smooth function of any set of parameters $\lambda$, or in the implicit form

$$
\boldsymbol{C}(\boldsymbol{p}, \boldsymbol{\tau})=\mathbf{0},
$$

where $\boldsymbol{C}(\boldsymbol{p}, \boldsymbol{\tau})$ is any smooth function with a full-rank Jacobian $\boldsymbol{C} \boldsymbol{p}, \boldsymbol{\tau}$. We show in [32] that, again, the resulting system of equations is suitable to the following continuation strategy.

\section{A Continuation Strategy}

To determine a path connecting $\boldsymbol{x}_{s}$ and $\boldsymbol{x}_{g}$ we rely on the higher-dimensional continuation method by Henderson [36], with the extensions proposed in [37] to heuristically guide the search towards $\boldsymbol{x}_{g}$. The approach provides a systematic way of marching through $\mathcal{M}$ from $\boldsymbol{x}_{s}$ until $\boldsymbol{x}_{g}$ has been found, or the entire connected component of $\boldsymbol{x}_{s}$ has been reached. The method is a generalisation of classical path-following techniques [38] and it is quite powerful as it can be applied to general smooth manifolds. It is only recently that its value is being recognized in the context of robotics $[32,39]$. We next recall its main points and refer the reader to $[36,37]$ for further details.

To explore $\mathcal{M}$, the method gradually grows an atlas on $\mathcal{M}$, i.e., a paving of $\mathcal{M}$ with flat tiles, or charts, that locally map regions of $\mathcal{M}$. At a given point $\boldsymbol{x}_{i} \in \mathcal{M}$, initially set to $\boldsymbol{x}_{s}$, the method computes the tangent space to $\mathcal{M}$ at $\boldsymbol{x}_{i}, \mathcal{T}_{i}$, and uses this space to
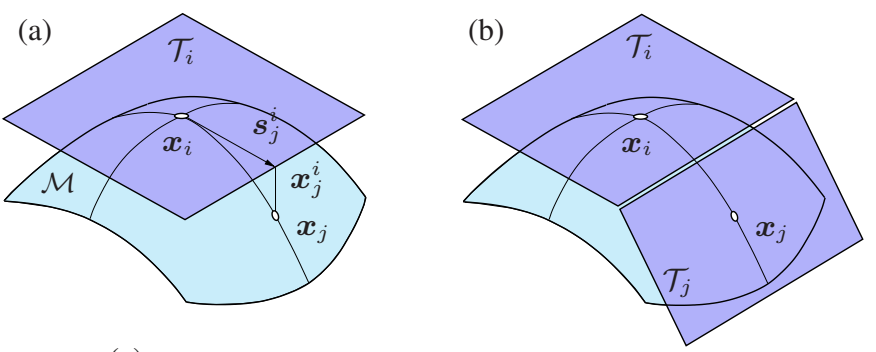

(c)
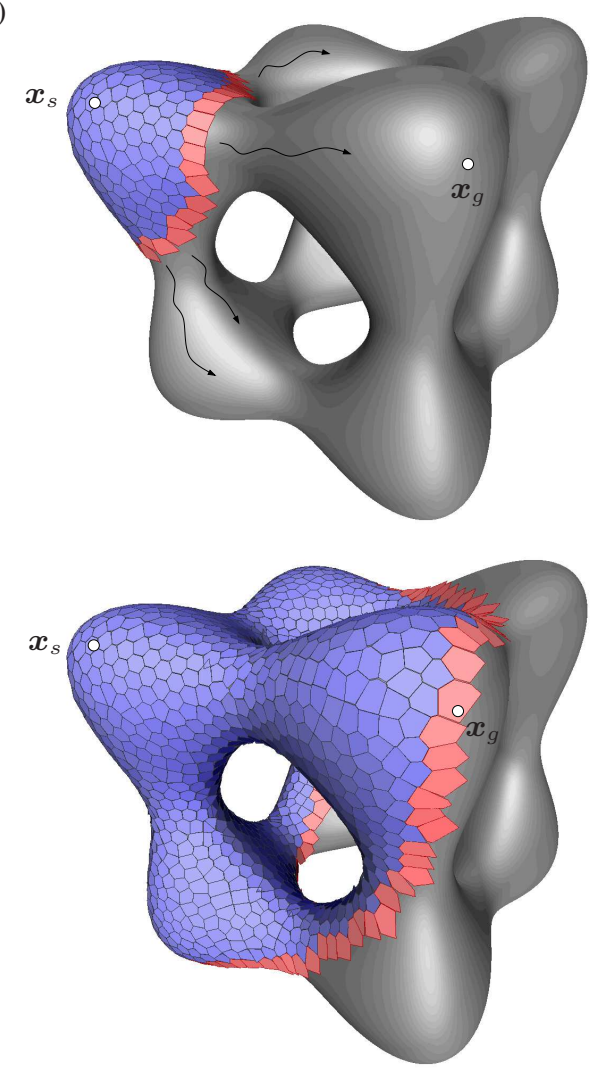

Fig. 5. (a) Chart construction around $\boldsymbol{x}_{i} \in \mathcal{M}$, and marching along direction $\boldsymbol{s}_{j}^{i}$ to produce a new point $\boldsymbol{x}_{j} \in \mathcal{M}$. (b) A neighboring chart is constructed at $x_{j}$, and it is properly clipped with existing charts. (c) Evolution of the continuation method on a Chmutov surface defined by $3+8\left(x^{4}+y^{4}+z^{4}\right)=8\left(x^{2}+y^{2}+z^{2}\right)$

chart the points in $\mathcal{M}$ around $\boldsymbol{x}_{i}$ [Fig. 5 (a)]. To continue the march, the method choses a direction $\boldsymbol{s}_{j}^{i} \in \mathcal{T}_{i}$, projects the point $\boldsymbol{x}_{j}^{i}$ down to $\mathcal{M}$ to obtain $\boldsymbol{x}_{j}$, and generates a new chart on $\mathcal{T}_{j}$, the tangent space to $\mathcal{M}$ at $\boldsymbol{x}_{j}$. The method keeps track of the regions of $\mathcal{M}$ explored up to a given point by clipping the chart domains on $\mathcal{T}_{i}$ and $\mathcal{T}_{j}$ against each other [Fig. 5 (b)], and the whole process is iterated until $\boldsymbol{x}_{g}$ is covered by some chart [Fig. 5 (c)], or the whole connected component reachable from $\boldsymbol{x}_{s}$ has been charted. The strategy has a mechanism to adapt the extension of the chart domains to the local curvature of the manifold at each point [36], and despite the atlas is discrete, it allows the generation of continuous paths from a chart center $\boldsymbol{x}_{i}$ to a neighboring one $\boldsymbol{x}_{j}$. To ensure the continuity of these paths, one can use multiprecision methods for example [40].

The expansion of the atlas may proceed in breadth-first order, as in Fig. 5 (c), it or may be guided towards $\boldsymbol{x}_{g}$ heuristically, as illustrated in Section $\mathrm{V}$ below. A possible approach is to use an $\mathrm{A}^{*}$ search strategy [41], which only generates the necessary charts to compute a minimum-cost path from $\boldsymbol{x}_{s}$ to $\boldsymbol{x}_{g}$. At each iteration, this method 
expands the chart of the point $\boldsymbol{x}_{i}$ yielding the lowest estimated cost of the whole movement path from $\boldsymbol{x}_{s}$ to $\boldsymbol{x}_{g}$, keeping a sorted priority queue of alternative path segments. The previous cost is the sum of a term $g\left(\boldsymbol{x}_{i}\right)$ that gives the lowest known cost of moving from $\boldsymbol{x}_{s}$ to $\boldsymbol{x}_{i}$, and a term $h\left(\boldsymbol{x}_{i}\right)$ that gives a lower bound of the cost of moving from $\boldsymbol{x}_{i}$ to $\boldsymbol{x}_{g}$. The value of $g\left(\boldsymbol{x}_{i}\right)$ is maintained during the expansion of the atlas by means of a function $c\left(\boldsymbol{x}_{j}, \boldsymbol{x}_{k}\right)$ that determines the transition cost between two chart centers. Examples of all of these functions are given in Section V.

The A* strategy typically performs well on manifolds of dimension up to three, but computation times considerably increase in larger dimensions. In such cases, one can simply use a Greedy Best-first strategy, in which the chart to be expanded is just the one yielding a minimum value $h\left(\boldsymbol{x}_{i}\right)$. In doing so, the path obtained may not be close to the optimal one, but the Greedy Best-first strategy usually generates less charts, and tends to be much faster. Both the $\mathrm{A}^{*}$ and Greedy Best-first strategies, however, will find a path on $\mathcal{M}$ from $\boldsymbol{x}_{s}$ to $\boldsymbol{x}_{g}$ whenever one exists.

During atlas construction, a graph $G$ can be built whose nodes represent the chart centers $\boldsymbol{x}_{i}$, and whose edges store the neighbouring relations between the computed charts. Thus, when $\boldsymbol{x}_{g}$ has been reached, we can use $G$ to rapidly generate a minimum-cost path connecting $\boldsymbol{x}_{s}$ with $\boldsymbol{x}_{g}$ according to the cost function assumed. Any cost function can be used in principle. Depending on the application, the function may reflect energy consumption, travel distance, or a penalty due to robot collisions with itself or with the environment. In the latter case the function only has to assign an infinite cost to the chart-to-chart transitions that are causing a collision [37]. Finally we note that, since the returned paths use direct motions between adjacent chart centers, they may be slightly jerky, but they can be smoothed using standard path smoothing techniques [42].

\section{Performance Tests}

We next illustrate the performance of the method on two instances of an octahedral hexapod, specified as Robot 1 and 2 hereafter (Fig. 6). The robots essentially have the structure of the NIST Robocrane [8], but the planner remains applicable to general hexapods, with cable anchor points not necessarily coincident in pairs.

In Section V-A we apply the planner to compute paths in twodimensional slices of $\mathcal{C}$ obtained by fixing four pose parameters. This shows how complex the wrench-feasible C-space can be even in simple cases, and stresses the advantages of our approach in comparison to previous methods based on discretization. Then, in Section V-B, we use the planner in a real prototype, both to plan motions subject to geometric constraints, and free-flying motions in six-dimensional space. Computation times are given in seconds in Table I at the end of the section.

All results have been obtained with an implementation in $\mathrm{C}$ of the method available as part of the open source package called CUIK [39], executed on a MacBook Pro computer equipped with a Intel Core i7 processor running at $2.66 \mathrm{GHz}$. Because of its attractive properties in parallel machines, the implementation adopts the tilt-and-torsion parameterization of $S O(3)$, for which $\boldsymbol{R}=\boldsymbol{R}_{z}(\phi) \boldsymbol{R}_{y}(\theta) \boldsymbol{R}_{z}(\sigma-\phi)$, where $\phi, \theta$, and $\sigma$ are the azimuth, tilt, and torsion angles respectively [28]. Thus, $\boldsymbol{\tau}=\{\phi, \theta, \sigma\}$ in this section, and the algorithms take into account that the angular coordinates differing in multiples of $2 \pi$ refer to the same angle.

\section{A. Planning in Illustrative Slices}

In this example, Robot 1 is required to withstand a load of $1 \mathrm{~N}$ applied at a point $P_{m}$ with position vector $\boldsymbol{p}_{m}=[30,14,-21]^{\top}$ $\mathrm{mm}$ in frame $\mathcal{F}_{2}$. Note that the weight of this load corresponds to a
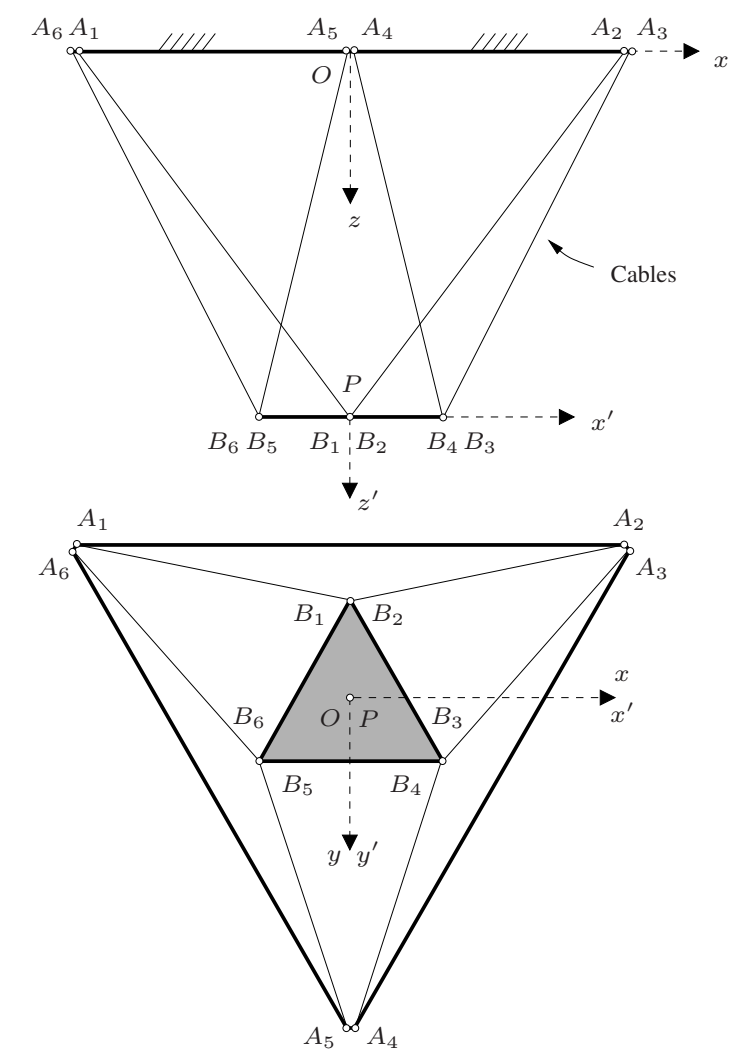

\begin{tabular}{|c|c|c|}
\hline & Base & Platform \\
\hline \multirow{6}{*}{ 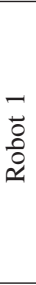 } & $A_{1}=(-200,-115.47,0)$ & $B_{1}=(0,-115.47,0)$ \\
\hline & $A_{2}=(200,-115.47,0)$ & $B_{2}=(0,-115.47,0)$ \\
\hline & $A_{3}=(200,-115.47,0)$ & $B_{3}=(100,57.74,0)$ \\
\hline & $A_{4}=(0,230.94,0)$ & $B_{4}=(100,57.74,0)$ \\
\hline & $A_{5}=(0,230.94,0)$ & $B_{5}=(-100,57.74,0)$ \\
\hline & $A_{6}=(-200,-115.47,0)$ & $B_{6}=(-100,57.74,0)$ \\
\hline \multirow{6}{*}{ 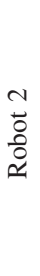 } & $A_{1}=(-231.62,-136.18,0)$ & $B_{1}=(0,-89.15,0)$ \\
\hline & $A_{2}=(231.62,-136.18,0)$ & $B_{2}=(0,-89.15,0)$ \\
\hline & $A_{3}=(233.74,-132.50,0)$ & $B_{3}=(77.21,44.57,0)$ \\
\hline & $A_{4}=(2.13,268.67,0)$ & $B_{4}=(77.21,44.57,0)$ \\
\hline & $A_{5}=(-2.13,268.67,0)$ & $B_{5}=(-77.21,44.57,0)$ \\
\hline & $A_{6}=(-233.74,-132.50,0)$ & $B_{6}=(-77.21,44.57,0)$ \\
\hline
\end{tabular}

Fig. 6. Top: Front and top views of the octahedral architecture in a reference configuration. Bottom: Coordinates of $A_{i}$ and $B_{i}$ in $m m$, expressed in $\mathcal{F}_{1}=O x y z$ and $\mathcal{F}_{2}=P x^{\prime} y^{\prime} z^{\prime}$ respectively, for the two robots considered.

constant wrench $\hat{\boldsymbol{w}}_{0}=[0,0,1,0,0,0]^{\top}$ (in SI units) if expressed in a frame $\mathcal{F}_{3}$ defined parallel to $\mathcal{F}_{1}$ and translating with $P_{m}$. The bounded perturbations of this wrench will be represented by the ellipsoid $\mathcal{K}$ centered in $\hat{\boldsymbol{w}}_{0}$ with $\boldsymbol{E}=10^{4} \boldsymbol{I}_{6}$, also expressed in $\mathcal{F}_{3}$. Both $\hat{\boldsymbol{w}}_{0}$ and $\boldsymbol{E}$ can be expressed in $\mathcal{F}_{1}$ using appropriate expressions provided in [32]. The tensions and lengths for all cables are constrained to $f_{i} \in(0.05,0.5) \mathrm{N}$ and $d_{i} \in(100,550) \mathrm{mm}$.

Fig. 7, top, shows two slices of the wrench feasible C-space of the robot, computed in Matlab using dense discretization for fixed values of $\boldsymbol{p}$ and $\sigma$. The configurations corresponding to $\mathcal{C}$ are indicated in green, while those that cannot be reached due to cable lengths or tensions out of range are represented by the orange and blue areas, respectively. The symmetries in the slices appear because $\{\phi, \theta, \sigma\}$ and $\{\phi+\pi,-\theta, \sigma\}$ represent the same orientation 


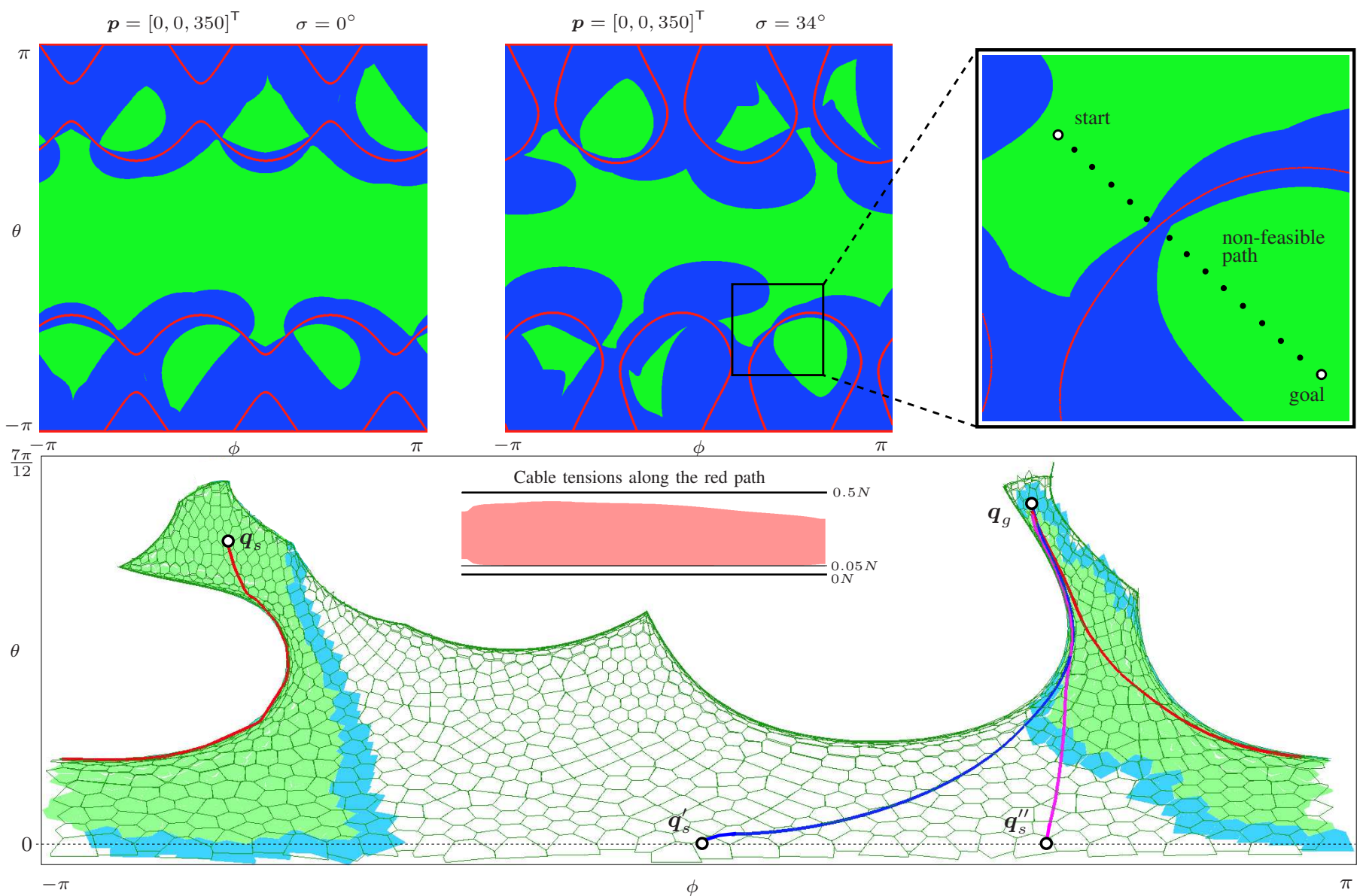

Fig. 7. Top: Slices of $\mathcal{C}$ for Robot 1 obtained by fixing $p$ and $\sigma$ at the values indicated. The images show the zones where some force is out of range (blue), some cable length exceeds its limits (orange), and configurations belonging to the wrench-feasible C-space (green). Note from the slices that the shape of $\mathcal{C}$ is intricate in general, and, in particular, that the path in the top-right picture is unfeasible, despite the wrench-feasibility conditions are satisfied when evaluated at the shown points. Bottom: Three paths computed by the proposed planner in the slice for $\boldsymbol{p}=[0,0,350]^{\top}$ and $\sigma=34^{\circ}$. The inset shows the envelope of cable tensions along the red path. See the text for details.

under the chosen parameterization. To avoid this double covering of $S O(3)$ we only need to restrict the expansion of the atlas to the range $\theta \in[0, \pi]$. The figures also show the singularity curves where $\operatorname{det}(\boldsymbol{J}(\boldsymbol{q}))=0$, in red, computed under no constraints on the cable tensions. It can be observed that, as expected, $\mathcal{C}$ naturally avoids crossing such curves, and that the navigation between two configurations is not trivial, because $\mathcal{C}$ is in general non-convex and may have very close connected components. The top-right plot of Fig. 7, in particular, exemplifies how the evaluation of the wrenchfeasibility conditions at discrete points only could result in erroneous paths linking configurations of $\mathcal{C}$ separated by singularity curves. In our case, because we rely on continuation, note that none of the paths computed by the proposed planner will misleadingly bridge two disjoint components of $\mathcal{C}$.

We next apply our method to resolve three planning queries on the slice for $\boldsymbol{p}=[0,0,350]^{\top}$ and $\sigma=34^{\circ}$, hence exploring a manifold of dimension $n=2$ (Fig. 7, bottom). Since here the platform can only rotate, we use the functions

$$
c\left(\boldsymbol{x}_{j}, \boldsymbol{x}_{k}\right)=\left\|\log \left(\boldsymbol{R}\left(\boldsymbol{\tau}_{j}\right)^{\top} \boldsymbol{R}\left(\boldsymbol{\tau}_{k}\right)\right)\right\|,
$$

and

$$
h\left(\boldsymbol{x}_{i}\right)=c\left(\boldsymbol{x}_{i}, \boldsymbol{x}_{g}\right) .
$$

to implement the A* search strategy described in Section IV. For two orientations given by $\boldsymbol{R}\left(\boldsymbol{\tau}_{j}\right)$ and $\boldsymbol{R}\left(\boldsymbol{\tau}_{k}\right), c\left(\boldsymbol{x}_{j}, \boldsymbol{x}_{k}\right)$ gives the angle of the axis-angle representation of $\boldsymbol{R}\left(\boldsymbol{\tau}_{j}\right)^{\top} \boldsymbol{R}\left(\boldsymbol{\tau}_{j}\right)$, which is a standard metric of $S O(3)$ [43].

In a first query, the start and goal configurations are given by the $\tau$ values $\left\{-2.3,1.6, \frac{17}{90} \pi\right\}$ rad and $\left\{1.7,1.7, \frac{17}{90} \pi\right\} \mathrm{rad}$, and by the position vector $\boldsymbol{p}=[0,0,350]^{\top} \mathrm{mm}$, yielding the points $\boldsymbol{q}_{s}$ and $\boldsymbol{q}_{g}$ shown in Fig. 7 bottom. The same figure depicts, in red, the path returned by the planner in this case, using the $A^{*}$ search strategy. In the figure, the green mesh corresponds to the full atlas of the connected component of $\mathcal{C}$ attainable from $\boldsymbol{q}_{s}$, and the shaded area corresponds to the part of this component that was actually explored by the A* method to connect $\boldsymbol{q}_{s}$ with $\boldsymbol{q}_{g}$. Green and blue charts respectively correspond to those lying in the interior and at the border of such area. It can be seen that, as expected, the algorithm biases the search towards $\boldsymbol{q}_{g}$, and how it correctly takes the topology of the angle variables into account. Moreover, notice from the figure that a naive approach based on simply following the rectilinear path from $\boldsymbol{q}_{s}$ to $\boldsymbol{q}_{g}$ would violate some of the constraints of $\mathcal{C}$, yielding uncontrollable motions or unaffordable cable tensions. The red path, in contrast, correctly avoids these situations and guarantees control of the platform at all points, keeping cable lengths and tensions within their allowable limits. The inset in Fig. 7 corroborates so, showing that the envelope of cable forces is admissible along the movement.

The line $\theta=0$ of Fig. 7, bottom, is known to be a representation singularity, because all of its points correspond to a same orienta- 
tion [28]. To illustrate that this is not a problem in practice, we issue two additional planning queries starting at distinct points of the $\theta=0$ line, $\boldsymbol{q}_{s}^{\prime}$ and $\boldsymbol{q}_{s}^{\prime \prime}$, both leading to $\boldsymbol{q}_{g}$. The paths returned by the A* planner, shown in blue and purple, are different, because the nature of the algorithm does not capture the fact that there is no cost of moving between two points with $\theta=0$. However, $\mathcal{M}$ is smooth despite the singularity, and the planner has no problem in computing feasible paths in both cases.

\section{B. Planning in a Real Prototype}

In order to mimic a situation in which the platform is subject to geometric constraints, we next apply our approach to the robot of Fig. 1, which is meant to perform insertion tasks on the surface of a sphere. For operation purposes, the platform is required to move tangentially to the sphere, with zero torsion. Using the parametric form of Eq. (14), these conditions can be written as follows

$$
\left.\begin{array}{l}
\boldsymbol{p}=\boldsymbol{r}_{c}+r_{s}\left[\begin{array}{c}
\cos \alpha_{2} \cos \alpha_{1} \\
\cos \alpha_{2} \sin \alpha_{1} \\
-\sin \alpha_{2}
\end{array}\right] \\
\phi=\alpha_{1}-\pi \\
\theta=\frac{\pi}{2}-\alpha_{2} \\
\sigma=0
\end{array}\right\},
$$

where $\boldsymbol{r}_{c}=\left[x_{s}, y_{s}, z_{s}\right]^{\top}$ and $r_{s}$ indicate the sphere center and radius, and $\alpha_{1}$ and $\alpha_{2}$ are two angular parameters. Thus, $\boldsymbol{\lambda}=\left\{\alpha_{1}, \alpha_{2}\right\}$ in this case, and the navigation manifold is of dimension $n=2$ after adding Eq. (16) to Eq. (13).

The points $A_{i}$ and $B_{i}$ are those of Robot 2 in Fig. 6, and the sphere is of radius $100 \mathrm{~mm}$, with its center located at $\boldsymbol{r}_{c}=[0,0,306]^{\top} \mathrm{mm}$ in frame $\mathcal{F}_{1}$. However, since a small distance between the platform and the sphere needs to be kept, a value of $r_{s}=130 \mathrm{~mm}$ is used in Eq. (16). The platform weight is of $0.6 \mathrm{~kg}$, with its center of mass located in $P$, and we use the same matrix $\boldsymbol{E}=10^{4} \boldsymbol{I}_{6}$ as before. Cable tensions are limited by the maximum force assumable by the motors, with $f_{i} \in(0.1,6.58) \mathrm{N}$, and the feasible lengths are those satisfying $d_{i} \in(200,600) \mathrm{mm}$, for $i=1, \ldots, 3$.

The resulting C-space is shown in Fig. 8 projected on the sphere, using the same drawing conventions of Fig. 7. The initial configuration, and the configurations where the insertion tasks are to be done are referred to as $\boldsymbol{q}_{1}, \boldsymbol{q}_{2}$, and $\boldsymbol{q}_{3}$, respectively, and correspond to the $\lambda$ values $\left\{0.55, \frac{\pi}{2}\right\},\{0.55,0.75\}$, and $\{2.63,0.75\}$. If we ask the planner to synthesize movements from $\boldsymbol{q}_{1}$ to $\boldsymbol{q}_{2}$, and then to $\boldsymbol{q}_{3}$, we obtain the red path in Fig. 8, which has been computed using

$$
c\left(\boldsymbol{x}_{i}, \boldsymbol{x}_{j}\right)=r_{s} \arctan \left(\frac{\left\|\boldsymbol{n}_{i} \times \boldsymbol{n}_{j}\right\|}{\boldsymbol{n}_{i} \cdot \boldsymbol{n}_{j}}\right)
$$

and

$$
h\left(\boldsymbol{x}_{i}\right)=c\left(\boldsymbol{x}_{i}, \boldsymbol{x}_{g}\right),
$$

in the A* search strategy, where $\boldsymbol{n}_{i}=\boldsymbol{p}_{i}-\boldsymbol{r}_{c}$. Given two points $\boldsymbol{p}_{i}$ and $\boldsymbol{p}_{j}$ on the sphere, these functions provide the great-circle distance between them, so the algorithm returns motions that minimize the distance travelled by $P$ on the surface of the sphere. A simple planning approach based on interpolation in the $\left\{\alpha_{1}, \alpha_{2}\right\}$ plane would result in a rather different motion. The transition from $\boldsymbol{q}_{1}$ to $\boldsymbol{q}_{2}$ would coincide, but the movement from $\boldsymbol{q}_{2}$ to $\boldsymbol{q}_{3}$ would yield the blue path of the figure, which rapidly leaves $\mathcal{C}$ at the beginning.

The video in https://youtu.be/GXSC9AQHLws shows the results of executing both the interpolated and planned versions of the $\boldsymbol{q}_{1}-\boldsymbol{q}_{2}-$ $\boldsymbol{q}_{3}$ movement. It can be seen how, as expected, the platform moves smoothly from $\boldsymbol{q}_{1}$ to $\boldsymbol{q}_{2}$, but some cables become slack and control of the platform is lost along the interpolated path from $\boldsymbol{q}_{2}$ to $\boldsymbol{q}_{3}$. Other undesirable effects include shakyness of the platform under small

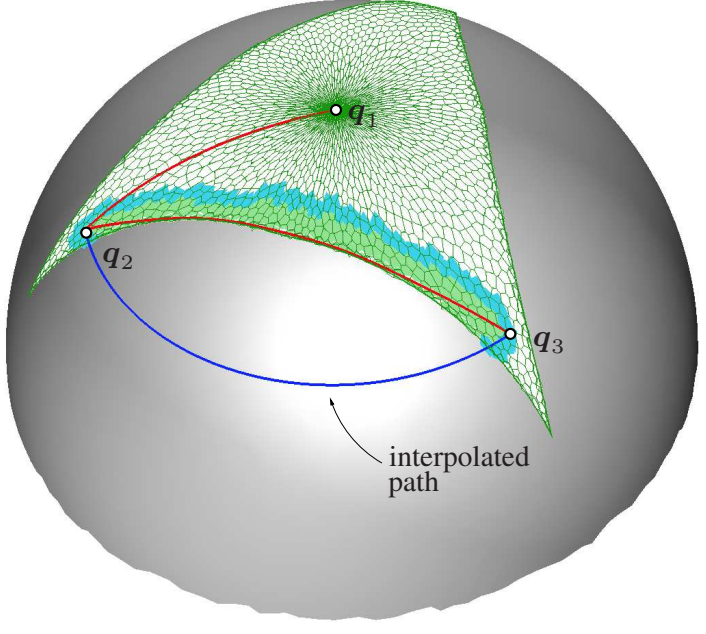

Fig. 8. Results of planning a path from $\boldsymbol{q}_{1}$ to $\boldsymbol{q}_{2}$, and then to $\boldsymbol{q}_{3}$, in the first experiment of Section V-B. The part of the C-space explored to plan the transition from $\boldsymbol{q}_{2}$ to $\boldsymbol{q}_{3}$ is shown shaded in green.

perturbations, collisions with the environment, and cable tanglement at the motors. In contrast, control of the platform is maintained when following the path $\boldsymbol{q}_{2}-\boldsymbol{q}_{3}$ returned by the planner. Fig. 9 summarizes the experiment in a few snapshots.

The method can be applied to higher-dimensional problems as well. For example, if the insertion operations are to be performed with an axisymmetric tool, we can ignore the zero-torsion constraint on the platform pose by removing $\sigma=0$ in Eq. (16). The result is a three-dimensional planning problem that is efficiently solved with the method, although the computation time is higher due to the increased size of the search space. Six-dimensional problems can also be solved by taking only Eq. (13) into account. Assuming that the sphere is not present, for example, a free-flying motion from $\boldsymbol{q}_{2}$ to $\boldsymbol{q}_{3}$ can rapidly be planned using the GBF strategy, using $h\left(\boldsymbol{x}_{i}\right)=\left\|\boldsymbol{x}_{i}-\boldsymbol{x}_{g}\right\|^{2}$.

The problem sizes and computation times of all test cases are reported in Table I, assuming the threshold values, defined in [37], $r=\varepsilon=0.15$. For each case, the table shows the dimension of the explored manifold $(n)$, the number of problem variables $(m)$, and the time spent by the Greedy Best-first and A* strategies (in seconds, last two columns), using the cost functions explained. As anticipated in Section IV, in terms of computation time the use of a Greedy Bestfirst strategy is advantageous in higher-dimensional problems, while the $\mathrm{A}^{*}$ one is affordable and adviseable in lower dimensions, because it normally yields lower-cost paths. Moreover, it must be said that once a partial atlas has been computed, all planning queries between configurations in such atlas can be solved in a few milliseconds.

\begin{tabular}{clccc} 
Robot & Path & $n / m$ & $\mathrm{GBF}$ & $\mathrm{A}^{*}$ \\
\hline \multirow{3}{*}{1} & $\boldsymbol{q}_{s} \rightarrow \boldsymbol{q}_{g}$ & $2 / 150$ & 29 & 62 \\
& $\boldsymbol{q}_{s}^{\prime} \rightarrow \boldsymbol{q}_{g}$ & $2 / 150$ & 6 & 12 \\
& $\boldsymbol{q}_{s}^{\prime \prime} \rightarrow \boldsymbol{q}_{g}$ & $2 / 150$ & 5 & 9 \\
\hline \multirow{3}{*}{2} & $\boldsymbol{q}_{1} \rightarrow \boldsymbol{q}_{2}$ & $2 / 155$ & 12 & 2 \\
& $\boldsymbol{q}_{2} \rightarrow \boldsymbol{q}_{3}$ & $2 / 155$ & 30 & 14 \\
& $\boldsymbol{q}_{2} \rightarrow \boldsymbol{q}_{3}$ ( $($ free) & $3 / 155$ & 56 & 166 \\
& $\boldsymbol{q}_{2} \rightarrow \boldsymbol{q}_{3}$ (free-flying) & $6 / 153$ & 40 & $>6000$ \\
\hline
\end{tabular}

TABLE I

PROBLEM SIZES AND COMPUTATION TIMES FOR ALL TEST CASES 

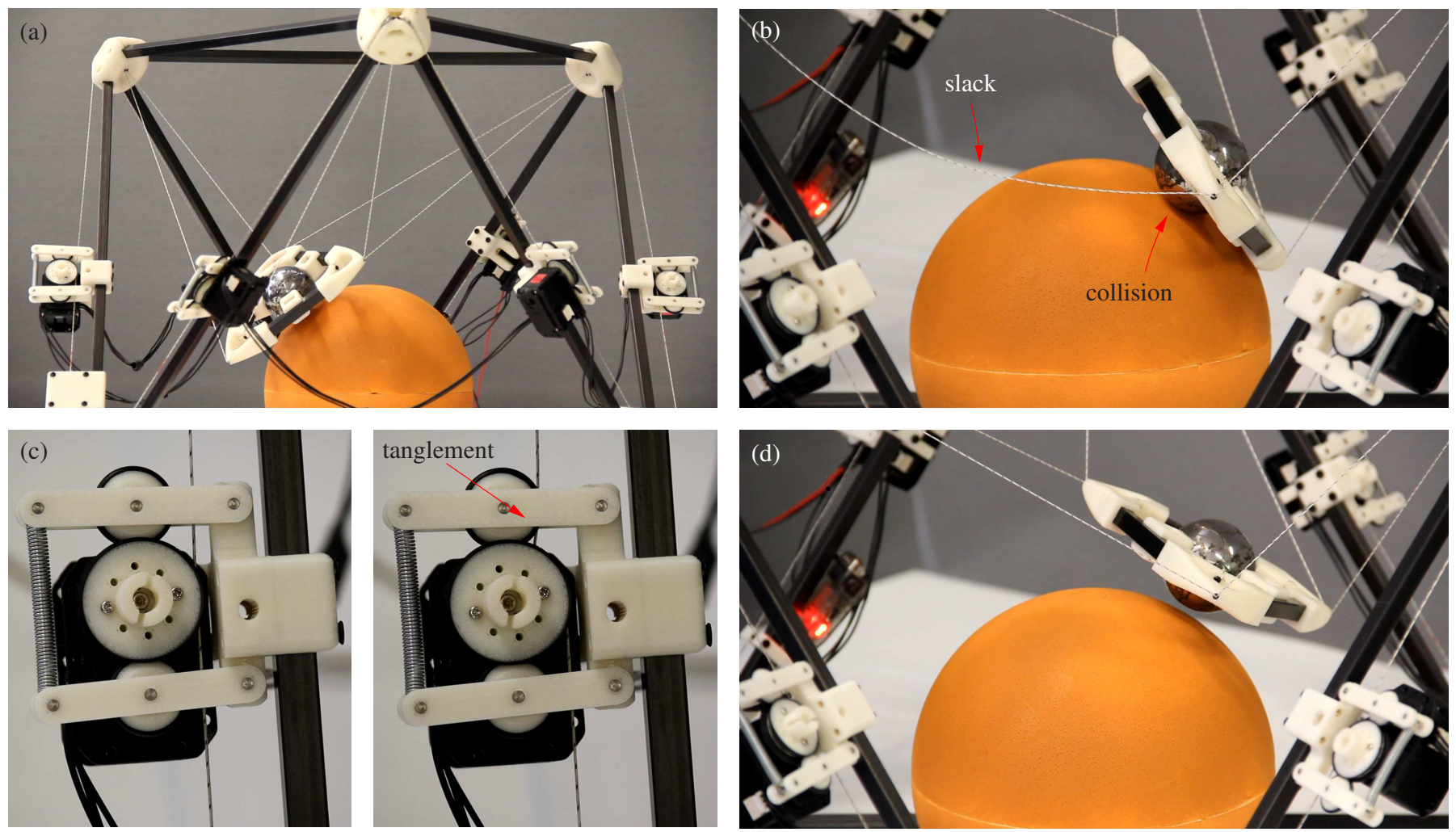

Fig. 9. Several snapshots of the video available in https://youtu.be/GXSC9AQHLws. (a): The robot at configuration $\boldsymbol{q}_{2}$. (b): Slackness of the cables and collisions appear when trying to follow the interpolated path from $\boldsymbol{q}_{2}$ to $\boldsymbol{q}_{3}$. (c): Comparison between normal operation (left) and tanglement of the cables in the motors (right). (d): Following the wrench-feasible path between $\boldsymbol{q}_{2}$ and $\boldsymbol{q}_{3}$ guarantees a smooth motion and control of the platform at all times.

\section{CONCLUSiOnS}

The ability to govern a load both in position and orientation is crucial in many applications, and parallel cable-driven robots constitute an advantageous, cost-effective solution. The problem is challenging because cable tensions need to be positive to avoid swaying and unwanted collisions of the load when moving towards a goal. This paper has proposed a path planner that ensures a safe navigation in this respect. The planner automatically computes motion paths that at any point allow the robot to counteract a platform wrench subject to bounded perturbations, with cable tensions lying inside their allowable bounds. As explained in the paper, such paths will never cross the forward singularity locus, and the adjustment of the tension bounds can be used to tune the clearance relative to the locus. When executing the planned paths, the load then moves smoothly and predictably towards the goal, which makes the approach suitable in fine manipulation tasks especially. Although the emphasis has been on modelling the length and tension constraints of the hexapod, the algorithm is flexible-enough to also accommodate collision constraints of the robot. As shown in Section IV, these simply translate into infinite cost transitions in the graph of the atlas. The method has been thoroughly tested in $\mathrm{C}$-spaces of various dimensions, and with experiments on a real prototype. Video sequences of the latter can be found in the multimedia material attached to the paper.

A number of points are proposed for future attention. First, in some applications it may be necessary to also obtain platform motions with a certain degree of position accuracy for the moving load. Due to the generality of the continuation strategy, it should be possible to deal with such constraints using by propagating known bounds on the position error of the actuators to an ellipsoidal bound on the platform pose. Second, while inertia effects can currently be modeled as bounded perturbations of the wrench, this approach is better suited to move the robot quasi-statically, as shown in our experiments. Further research needs to be done to see whether the method can be extended to also synthesize motion paths ensuring a full dynamic control of the robot, or even a time-optimal trajectory. As noted in [44], substantial workspace enlargements should be achieved in doing so. Third, since the robot geometry is also subject to uncertainty, it would be adviseable to develop a local planner able to take such uncertainties into account in the transitions between chart centers, translating the developments in [45] to the cable-driven context, for example. Finally, efforts should also be made to extend the method to deal with overactuated robots with somewhat elastic cables, which, despite their more intrusive nature, are increasingly proposed to exploit their redundant actuation [46].

\section{ACKNOWLEDGEMENTS}

The authors wish to express their gratitude to M. E. Henderson for introducing them to higher-dimensional continuation, to Patrick Grosch for constructing the robot prototype used in the paper, and to Josep M. Porta, for valuable discussions on the topic of the paper, and for his help on the algorithms of the CUIK suite.

\section{REFERENCES}

[1] P. Cheng, J. Fink, S. Kim, and V. Kumar, "Cooperative towing with multiple robots," in Algorithmic Foundations of Robotics VIII, ser. Springer Tracts in Advanced Robotics, G. Chirikjian, H. Choset, M. Morales, and T. Murphey, Eds. Springer Berlin / Heidelberg, 2009, vol. 57, pp. 101-116.

[2] S. R. Oh, J. C. Ryu, and S. K. Agrawal, "Dynamics and control of a helicopter carrying a payload using a cable-suspended robot," Journal of Mechanical Design, vol. 128, no. 5, pp. 1113-1121, 2006. 
[3] A. M. Lytle, K. S. Saidi, R. V. Bostelman, W. C. Stone, and N. A. Scott, "Adapting a teleoperated device for autonomous control using three-dimensional positioning sensors: experiences with the NIST RoboCrane," Automation in Construction, vol. 13, pp. 101-118, 2004.

[4] P. H. Borgstrom, B. L. Jordan, B. J. Borgstrom, M. J. Stealey, G. S. Sukhatme, M. A. Batalin, and W. J. Kaiser, "NIMS-PL: A cabledriven robot with self-calibration capabilities," IEEE Trans. on Robotics, vol. 25, no. 5, pp. $1005-1015,2009$.

[5] P. Bosscher, R. L. Williams II, L. S. Bryson, and D. Castro-Lacouture, "Cable-suspended robotic contour crafting system," Automation in Construction, vol. 17, pp. 45-55, 2007.

[6] J.-P. Merlet and D. Daney, "A portable, modular parallel wire crane for rescue operations," in Proc. of the IEEE Intl. Conference on Robotics and Automation, 2010, pp. $2834-2839$.

[7] S. Tadokoro, T. Matsushima, Y. Murao, and H. Kohkawa, "A parallel cable-driven motion base for virtual acceleration," in Proc. of the IEEE/RSJ Intl. Conference on Intelligent Robots and Systems, vol. 3, 2001, pp. $1700-1705$

[8] J. Albus, R. V. Bostelman, and N. Dagalakis, "The NIST Robocrane," Journal of Robotic Systems, vol. 10, no. 5, pp. 709-724, 1993.

[9] R. Bostelman, J. Albus, N. Dagalakis, A. Jacoff, and J. Gross, “Applications of the NIST RoboCrane," in Proc. of the 5th Intl. Symposium on Robotics and Manufacturing, 1994, pp. 14-18.

[10] A. M. Lytle and K. S. Saidi, "NIST research in autonomous construction," Autonomous Robots, vol. 22, no. 3, pp. 211-221, 2007.

[11] P. Bosscher, A. T. Riechel, and I. Ebert-Uphoff, "Wrench-feasible workspace generation for cable-driven robots," IEEE Trans. on Robotics, vol. 22, no. 5, pp. 890-902, 2006.

[12] J.-P. Merlet, "Checking the cable configuration of cable-driven parallel robots on a trajectory," in Robotics and Automation (ICRA), 2014 IEEE Intl. Conference on. IEEE, 2014, pp. 1586-1591.

[13] R. Verhoeven, "Analysis of the workspace of tendon based Stewart platforms," Ph.D. dissertation, Universität Duisburg-Essen, 2004

[14] I. Ebert-Uphoff and P. Voglewede, "On the connections between cabledriven robots, parallel robots and grasping," in Proc. of the IEEE Intl. Conference on Robotics and Automation, 2004, pp. 4521-4526.

[15] P. Bosscher, A. Riechel, and I. Ebert-Uphoff, "Wrench-feasible workspace generation for cable-driven robots," IEEE Trans. on Robotics, vol. 22, no. 5, pp. 890-902, 2006.

[16] J. Pusey, A. Fattah, S. K. Agrawal, and E. Messina, "Design and workspace analysis of a 6-6 cable-suspended parallel robot," Mechanism and Machine Theory, vol. 3, pp. 761-778, 2004

[17] E. Stump and V. Kumar, "Workspaces of cable-actuated parallel manipulators," ASME Journal of Mechanical Design, vol. 128, no. 1, pp. 159-167, 2006.

[18] M. Gouttefarde, D. Daney, and J.-P. Merlet, "Interval-analysis-based determination of the wrench-feasible workspace of parallel cable-driven robots," IEEE Trans. on Robotics, vol. 27, no. 1, pp. 1-13, 2011.

[19] S. Bhattacharya, H. Hatwal, and A. Ghosh, "Comparison of an exact and an approximate method of singularity avoidance in platform type parallel manipulators," Mechanism and Machine Theory, vol. 33, no. 7, pp. 965-974, 1998.

[20] B. Dasgupta and T. Mruthyunjaya, "Singularity-free path planning for the Stewart platform manipulator," Mechanism and Machine Theory, vol. 33, no. 6, pp. 711-725, 1998.

[21] S. Sen, B. Dasgupta, and A. K. Mallik, "Variational approach for singularity-free path-planning of parallel manipulators," Mechanism and Machine Theory, vol. 38, no. 11, pp. 1165-1183, 2003.

[22] A. K. Dash, I.-M. Chen, S. H. Yeo, and G. Yang, "Workspace generation and planning singularity-free path for parallel manipulators," Mechanism and Machine Theory, vol. 40, no. 7, pp. 776-805, 2005.

[23] P. Voglewede and I. Ebert-Uphoff, "Overarching framework for measuring closeness to singularities of parallel manipulators," IEEE Trans. on Robotics, vol. 21, no. 6, pp. 1037-1045, 2005.

[24] S. Fang, D. Franitza, R. Verhoeven, and M. Hiller, "Optimum motion planning for tendon-based Stewart platforms," in Proc. of the 11th IFToMM World Congress in Mechanism and Machine Science, H. Tian, Ed. Tianjin, China: China Machinery Press, 2003.

[25] M. Hiller, S. Fang, S. Mielczarek, R. Verhoeven, and D. Franitza, "Design, analysis and realization of tendon-based parallel manipulators," Mechanism and Machine Theory, vol. 40, no. 4, pp. 429-445, 2005.

[26] S. Lahouar, E. Ottaviano, S. Zeghoul, L. Romdhane, and M. Ceccarelli, "Collision free path-planning for cable-driven parallel robots," Robotics and Autonomous Systems, vol. 57, no. 11, pp. 1083-1093, 2009.

[27] O. Bohigas, M. Manubens, and L. Ros, "Navigating the wrench-feasible C-space of cable-driven hexapods," in Cable-Driven Parallel Robots, T. Bruckmann and A. Pott, Eds. Springer, 2012, pp. 53-68.
[28] I. A. Bonev, D. Zlatanov, and C. M. Gosselin, "Advantages of the modified Euler angles in the design and control of PKMs," in Proc. of the 3rd Chemnitz Parallel Kinematics Seminar/2002 Parallel Kinematic Machines Intl. Conference, Chemnitz, Germany, 2002, pp. 171-188.

[29] P. Corke, Robotics, Vision and Control: Fundamental Algorithms in MATLAB, ser. Springer Tracts in Advanced Robotics. Springer, 2011, vol. 73.

[30] K. J. Waldron and K. H. Hunt, "Series-parallel dualities in actively coordinated mechanisms," Intl. Journal of Robotics Research, vol. 10, pp. 473-480, 1991.

[31] L. Ros, A. Sabater, and F. Thomas, "An ellipsoidal calculus based on propagation and fusion," IEEE Trans. on Systems, Man, and Cybernetics, Part B, vol. 32, no. 4, pp. 430-442, 2002.

[32] O. Bohigas, M. Manubens, and L. Ros, Singularities of Robot Mechanisms: Numerical Computation and Avoidance Path Planning, ser. Mechanism and Machine Science. Springer, in press.

[33] M. E. Henderson, Numerical Continuation Methods for Dynamical Systems. Springer, 2007, ch. Higher-Dimensional Continuation, pp. $77-115$.

[34] O. Bohigas, "Numerical computation and avoidance of manipulator singularities," Ph.D. dissertation, Universitat Politècnica de Catalunya, 2013, available through the web address http://goo.gl/wlR0i.

[35] M. E. Henderson, "Multiparameter parallel search branch switching," Intl. Journal of Bifurcation and Chaos in Applied Science and Engineering, vol. 15, no. 3, pp. 967-974, 2005.

[36] — , "Multiple parameter continuation: Computing implicitly defined k-manifolds," Intl. Journal of Bifurcation and Chaos, vol. 12, no. 3, pp. 451-476, 2002.

[37] O. Bohigas, M. Henderson, L. Ros, M. Manubens, and J. Porta, "Planning singularity-free paths on closed-chain manipulators," Robotics, IEEE Trans. on, vol. 29, no. 4, pp. 888-898, Aug 2013.

[38] E. Allgower and K. Georg, "Numerical path following," Handbook of Numerical Analysis, vol. 5, no. 3, pp. 3-207, 1997.

[39] J. Porta, L. Ros, O. Bohigas, M. Manubens, C. Rosales, and L. Jaillet, "The CUIK suite: Motion analysis of closed-chain multibody systems," IEEE Robotics and Automation Magazine, vol. 21, no. 3, pp. 105-114, September 2014

[40] D. J. Bates, J. D. Hauenstein, A. J. Sommese, and C. W. Wampler, "Adaptive multiprecision path tracking," SIAM Journal on Numerical Analysis, vol. 46, no. 2, pp. 722-746, 2008.

[41] S. J. Russell and P. Norvig, Artificial Intelligence: A Modern Approach. Prentice Hall, 2003.

[42] D. Berenson, S. S. Srinivasa, and J. Kuffner, "Task space regions: A framework for pose-constrained manipulation planning," The Intl. Journal of Robotics Research, vol. 30, no. 12, pp. 1435-1460, Oct 2011.

[43] D. Q. Huynh, "Metrics for 3D rotations: Comparison and analysis," Journal of Mathematical Imaging and Vision, vol. 35, no. 2, pp. 155164,2009

[44] C. Gosselin, P. Ren, and S. Foucault, "Dynamic trajectory planning of a two-DOF cable-suspended parallel robot," in Robotics and Automation (ICRA), 2012 IEEE Intl. Conference on, May 2012, pp. 1476-1481.

[45] J.-P. Merlet, "A local motion planner for closed-loop robots," in Intelligent Robots and Systems, 2007. IROS 2007. IEEE/RSJ Intl. Conference on. IEEE, 2007, pp. 3088-3093.

[46] S.-R. Oh and S. K. Agrawal, "Cable suspended planar robots with redundant cables: controllers with positive tensions," Robotics, IEEE Transactions on, vol. 21, no. 3, pp. 457-465, 2005 